\title{
Inclusão de aditivos probióticos em dietas para tilápia do Nilo (Oreochromis niloticus) e seus efeitos no desempenho, parâmetros hematológicos, hepáticos e intestinais
}

\author{
Inclusion of probiotic additives in diets for Nile tilapia (Oreochromis niloticus) and its effects on \\ performance, hematological, hepatic and intestinal parameters \\ Inclusión de aditivos probióticos en dietas de tilapia del Nilo (Oreochromis niloticus) y sus efectos \\ sobre el rendimiento, parámetros hematológicos, hepáticos e intestinales
}

\begin{abstract}
Resumo
Objetivo deste trabalho foi avaliar a inclusão de dois tipos de probiótico comercial, um complexo de bactérias e outro com Bacillus Subtillis em dietas para juvenis de tilápia do Nilo. Foram investigados os efeitos no desemprenho zootécnico, parâmetros hematológicos, concentração de glicose, morfologia intestinais e histomorfometria dos

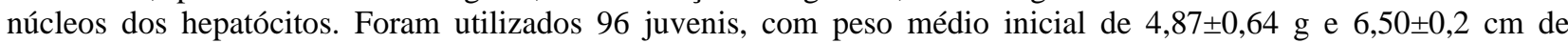
comprimento total, distribuídos aleatoriamente em 12 tanques de $18 \mathrm{~L}$, com oito peixes em cada unidade experimental durante 53 dias, com diferentes tratamentos: (C) Controle; (CB) ração com complexo de bactérias; (BS) ração com $B$. subtilis. Entre os tratamentos não apresentaram diferença significativa no desempenho zootécnico da tilápia do Nilo. Para os parâmetros hematológicos, verificou-se diferença entre os tratamentos no número de eritrócitos, onde os peixes tratados com complexo de bactérias tiveram os maiores valores em relação aos grupos controle e $B$. subtilis, porém não teve alteração para os outros parâmetros sanguíneos. Na análise da composição centesimal não foi observado diferença significativa entre os tratamentos. As vilosidades intestinais foram maiores para CB em altura e largura dos vilos, porém a espessura da túnica foi maior no tratamento BS. A histomorfométrica dos núcleos dos
\end{abstract}


hepatócitos os peixes tratados com CB tiveram maiores valores para área $\left(\mu \mathrm{m}^{2}\right)$, perímetro $(\mu \mathrm{m})$ e volume $\left(\mu \mathrm{m}^{3}\right)$ dos núcleos. De acordo com os dados coletados, não há diferenças significativas entre aplicação de complexo de bactérias e B. subtilis.

Palavras-chave: Bactérias; Centesimal; Histologia; Vilosidade.

\begin{abstract}
The aim of this study was to evaluate the inclusion of two commercial probiotics, a bacterial complex and a complex containing Bacillus subtilis in diets for Nile tilapia juveniles. The effects on growth performance, hematological parameters, glucose concentration, intestinal morphology and histomorphometry of hepatocytes' nuclei were assessed. Ninety-six fish juveniles with mean initial weight of $4.87 \pm 0.64 \mathrm{~g}$ and $6.50 \pm 0.2 \mathrm{~cm}$ total length were randomly distributed in 12 tanks $(18 \mathrm{~L})$, with eight fish in each tank, during 53 days. The following treatments were designed: (C) control, (CB) feed containing the bacterial complex, and (BS) feed containing B. subtilis. No significant differences were found in relation to the growth performance of fish. In relation to the hematological parameters, a single difference was observed regarding the number of erythrocytes, with the fish treated with the diet containing the bacterial complex displayed the highest values in relation to the C and BS groups, while the other parameters were not affected by the probiotic inclusions. Similarly, no differences were observed for the proximate composition of fish. The intestinal villi of fish fed the bacterial complex (CB) were higher and wider, but the thickness of the tunic was higher in B. subtilis. The histomorphometric variables of the hepatocytes' nuclei of the fish from the CB treatment revealed higher values of area $\left(\mu \mathrm{m}^{2}\right)$, perimeter $(\mu \mathrm{m})$ and volume $(\mu \mathrm{m} 3)$. It was concluded that there are no significant differences between the application of a bacterial complex or B. subtilis as probiotic additives for Nile tilapia juveniles.
\end{abstract}

Keywords: Bacteria; Proximate; Histology; Villus.

\title{
Resumen
}

El objetivo de este trabajo fue evaluar la inclusión de dos tipos de probiótico comercial, un complejo bacteriano y un complejo de Bacillus Subtillis en dietas para juveniles de tilapia del Nilo. Se investigaron los efectos sobre el rendimiento zootécnico, los parámetros hematológicos, la concentración de glucosa, la morfología intestinales y la histomorfometría de los núcleos de los hepatocitos. Se utilizaron 96 juveniles, con un peso inicial promedio de $4.87 \pm$ 0.64 gy $6.50 \pm 0.2 \mathrm{~cm}$ de tamaño total, distribuidos aleatoriamente en 12 tanques de $18 \mathrm{~L}$, con ocho peces en cada unidad experimental durante 53 días, con diferentes tratamientos: (C) Control; (CB) dieta con complejo bacteriano; (BS) ración de $B$. subtilis. No hubo diferencias significativas entre tratamientos en el desempeño zootécnico de la tilapia del Nilo. Para los parámetros hematológicos, hubo una diferencia entre los tratamientos en el número de eritrocitos, donde los peces tratados con el complejo bacteriano tuvieron los valores más altos en comparación con los grupos control y B. subtilis, pero no hubo cambios para los otros parámetros sanguíneos. En el análisis de composición centesimal, no se observó diferencia significativa entre tratamientos. Las vellosidades intestinales fueron mayores para $\mathrm{CB}$ en altura y ancho de las vellosidades, pero el grosor de la túnica fue mayor en el tratamiento BS. En el análisis histomorfométrico de núcleos de hepatocitos, los peces tratados con CB presentaron mayores valores de área $\left(\mu \mathrm{m}^{2}\right)$, perímetro $(\mu \mathrm{m})$ y volumen $\left(\mu \mathrm{m}^{3}\right)$ de los núcleos. Según los datos recopilados, no existen diferencias significativas entre la aplicación del complejo bacteriano y B. subtilis.

Palabras clave: Bacteria; Centesimal; Histología; Vellosidad.

\section{Introdução}

A produção de pescado no Brasil por meio da aquicultura vem aumentando a cada ano, sobrepujando outros setores de produção de alimento de origem animal, sendo uma forma de gerar renda, emprego, segurança alimentar para população e sustentabilidade. Segundo a Organização das Nações Unidas para a Alimentação e a Agricultura-FAO (2018) a tilápia do Nilo (Oreochromis niloticus) é a quarta espécie mais produzida no mundo, devido o vasto conhecimento sobre sua biologia e o avanço nas novas tecnologia desenvolvida para criação de peixes. No Brasil os principais produtores de pescado são os estados do Paraná com 166.000 t, São Paulo 70.500 t, Minas Gerais 42.100 t, Santa Catarina 40.059 t e Mato Grosso do Sul 29.090 t (PEIXE BR 2021). Estes estados vêm crescendo na produção de alimento e se destacando no território nacional (IBGE, 2017). Entre estes estados o Paraná é principal produtor de tilápia do Nilo, principalmente na sua região do oeste do Paraná, onde se concentra a maior produção do país (Schulter \& Vieira Filho, 2017; IBGE, 2018).

Com a intensificação do setor aquícola agregado aos problemas sanitários, proporcionou o surgimento de doenças, ocasionando o uso antibióticos para combater as enfermidades e melhorar a produção. Contudo, alguns microrganismos ficarão resistente a estes antibióticos, por falta de conhecimento técnico para uso ideal e/ou indiscriminado dos fármacos (Da Costa et 
al., 2008; Amarante et al.,2018; Khati et al., 2018). Em contrapartida ao surgimento destes problemas, o desenvolvimento de estudos com uso de aditivos probióticos mostrou-se importante para aquicultura. Atualmente na criação de peixes o uso de bactérias está desempenhando papel importante no desenvolvimento e metabolismo dos peixes (Ribeiro et al., 2008; Rego et al.,2012; Ferreira et al., 2015; Ibrahem, 2015; Fonseca et al 2020).

Os probióticos agem no desempenho dos peixes aumentando a digestão dos alimentos, diminuindo o estresse em sistema de alta densidade e melhorando o sistema imunológico, além disso, reduz a quantidade de composto nitrogenados e fosforo da água de cultivo (Ibrahem, 2015; Fonseca et al. 2020). Porém, no mercado existem vários produtos de probiótico, podendo ser comercializados em forma de líquido ou em pó, contendo uma bactéria ou várias bactérias em sua composição. No entanto, os dois tipos são usados na criação de peixes devido a seus pontos positivos. O produto líquido é mais fácil para o piscicultor incorporar na ração, e na água de cultivo como biorremediador (Van Hai, 2015), enquanto, que o produto em pó precisa de mais técnica para misturar o probiótico na ração, pois precisa utilizar uma porcentagem de $2 \%$ a 3\% óleo vegetal ou animal em relação com o peso da ração, contudo, as duas formas de apresentação do produto precisam de orientação técnica (Carvalho et al., 2011; Nakandakare et al., 2013; Fonseca et al. 2020). Vale ressaltar que segundo (Brito et al.,2014) ainda não existe um tipo de composição ideal de probiótico.

O presente trabalho teve como objetivo avaliar os efeitos do uso de dois tipos de probiótico comercial, um complexo de bactérias e o outro contendo apenas Bacillus Subtillis, no desempenho zootécnico, alterações hematológicas, intestinais e hepáticas em de juvenis de tilápia do Nilo.

\section{Metodologia}

\subsection{Material biológico e procedimentos}

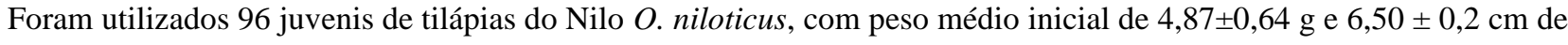
comprimento total, distribuídos aleatoriamente em 12 aquários com capacidade de $18 \mathrm{~L}$ e com oito peixes em cada unidade experimental, com renovação de água constante.

O experimento foi realizado no laboratório de Aquicultura da Universidade Estadual do Oeste do Paraná (Unioeste) Campus de Toledo-PR, durante o período de 53 dias. O sistema utilizado mantinha um fluxo de água sem sua reutilização, com fonte de oxigenação continua promovida por um soprador de ar central, o qual permaneceu conectado por mangueiras e pedras porosas em cada aquário.

A alimentação dos peixes foi realizada quatro vezes ao dia até saciedade aparente, com ração comercial extrusada (tamanho $2,0 \mathrm{~mm}$ e $35 \%$ de proteína bruta), no qual os peixes foram submetidos aos seguintes regimes alimentares: tratamentos controle (C) não contendo probiótico; (CB) ração com complexo de bactérias; (BS) ração com probiótico $B$. subtilis. O delineamento experimental foi inteiramente casualizado e composto por três tratamentos e quatro repetições.

A composição da ração fornecida no rotulo de fabricante foi: farelo de milho desengordurado, milho integral moído, farelo de soja, farelo de trigo, farinha de carne e ossos, calcário calcítico, cloreto de sódio (sal comum), metionina, lisina, antifúngico, premix vitamínico e mineral. Na tabela 1 encontram-se os níveis de garantia da ração comercial. 
Tabela 1. Níveis de garantia apresentado no rótulo da ração comercial.

\begin{tabular}{llll}
\hline & & Níveis de Garantia & \\
\hline Umidade & $120,0 \mathrm{~g} / \mathrm{kg}$ & Vitamina B6 & $6,0 \mathrm{mg} / \mathrm{kg}$ \\
Proté́na bruta & $350,0 \mathrm{~g} / \mathrm{kg}$ & Vitamina B12 & $10,0 \mu \mathrm{g} / \mathrm{kg}$ \\
Extrato etéreo & $50,0 \mathrm{~g} / \mathrm{kg}$ & Biotina & $0,5 \mathrm{mg} / \mathrm{kg}$ \\
Matéria fibrosa & $50,0 \mathrm{~g} / \mathrm{kg}$ & Antioxidante & $120,0 \mathrm{mg} / \mathrm{kg}$ \\
Matéria mineral & $80,0 \mathrm{~g} / \mathrm{kg}$ & Ác. Nitcotínico & $30,0 \mathrm{mg} / \mathrm{kg}$ \\
*Energia bruta & $4.127,5 \mathrm{kcal} / \mathrm{kg}$ & Ác. Pantotênico & $10,0 \mathrm{mg} / \mathrm{kg}$ \\
Calcio (mínimo) & $10,5 \mathrm{~g} / \mathrm{kg}$ & Ác.Fólico & $0,5 \mathrm{mg} / \mathrm{kg}$ \\
Calcio (máximo) & $15,0 \mathrm{~g} / \mathrm{kg}$ & Ferro & $40,0 \mathrm{mg} / \mathrm{kg}$ \\
Fosforo & $5.000,0 \mathrm{mg} / \mathrm{kg}$ & Cobre & $8,0 \mathrm{mg} / \mathrm{kg}$ \\
Vitamina C & $450,0 \mathrm{mg} / \mathrm{kg}$ & Manganês & $70,0 \mathrm{mg} / \mathrm{kg}$ \\
Vitamina A & $8.000,0 \mathrm{Ul} / \mathrm{kg}$ & Zinco & $50,0 \mathrm{mg} / \mathrm{kg}$ \\
Vitamina D3 & $2.100,0 \mathrm{Ul} / \mathrm{kg}$ & Iodo & $1,2 \mathrm{mg} / \mathrm{kg}$ \\
Vitamina E & $100,0 \mathrm{Ul} / \mathrm{kg}$ & Selênio & $0,12 \mathrm{mg} / \mathrm{kg}$ \\
Vitamina K3 & $3,0 \mathrm{mg} / \mathrm{kg}$ & Colina & $500,0 \mathrm{mg} / \mathrm{kg}$ \\
Vitamina B1 & $2,0 \mathrm{mg} / \mathrm{kg}$ & & \\
Vitamina B2 & $4 \mathrm{mg} / \mathrm{kg}$ & & \\
\hline
\end{tabular}

* Energia bruta calculada de acordo com o valor do calor de combustão de 9,44, 4,11 e 5,64 kcal g-1 para lipídios, carboidratos e proteínas, respectivamente (Braxter, 1989). Fonte: Kowalski: ração para peixes.

Foram administrados dois tipos de probióticos comerciais, os quais foram adquiridos em pó (liofilizado), e continha um complexo de bactérias (B. subtilis, B. licheniformis, B. amyloliquefaciens, B. cereus e Lactococcus lactis) na concentração de $1,25 \times 10^{8} \mathrm{UFC}^{-1}$. Efetivou-se o procedimento para a inclusão nas raçoes experimentais pelo método de aspersão, misturando-se em 3\% de óleo de soja referente ao peso da dieta, para melhorar a estabilidade. Enquanto o probiótico comercial líquido, apresentava em sua composição a bactéria (B. subtilis) na concentração de 2,0x 10 ${ }^{8} \mathrm{UFC}_{\text {.mL }}{ }^{-1}$, sendo acrescentada em um teor de $2,5 \mathrm{~mL} \cdot \mathrm{kg}^{-1}$ da ração experimental, conforme recomendado pelos fabricantes. Ambas foram homogeneizada por 10 minutos.

Foram avaliados diariamente as variáveis da temperatura $\left({ }^{\circ} \mathrm{C}\right)$ da água foi diariamente no período vespertino e os parâmetros pH, oxigênio dissolvido $\left(\mathrm{mg} . \mathrm{L}^{-1}\right)$ e condutividade elétrica $\left(\mu \mathrm{S} . \mathrm{cm}^{-1}\right)$ da água e sólidos totais dissolvidos $\left(\mathrm{mg} . \mathrm{L}^{-1}\right)$, que foram mensurados com um multiparametro (Hanna HI98196), sempre antes do sifonamento dos tanques (Sibaúba-Tavares 1995; Dias et al., 2011).

No início e final do experimento foi realizada a biometria para obtenção das medidas individuais de peso e comprimento total dos peixes com um paquímetro e uma balança de precisão $(0,001 \mathrm{~g})$. Posteriormente foram empregadas no cálculo e avaliação das variáveis zootécnicas. Foram determinados por: Sobrevivência $(\mathrm{SO})=\left(\mathrm{n}^{\circ}\right.$ de peixes no final/número de peixes inicial) x 100; Ganho de peso $(\mathrm{GP})=$ peso final - peso inicial; Eficiência alimentar $(\mathrm{EA})=(\mathrm{ganho}$ de peso(g)/consumo de ração $(\mathrm{g})$ ) /100; Taxa de crescimento específico $(\mathrm{TCE})=(\ln$ peso final $-\ln$ peso inicial $) /$ tempo de experimento $) \mathrm{x} 100$; Índice viscerosomático $($ IVS $)=($ peso das vísceras/peso corporal) $\mathrm{x} 100$; Índice hepatossomático $(\mathrm{IHS})=($ peso do fígado/ peso corporal) x100 (Silva \& Queiroz 2002).

\subsection{Análise centesimal}

A composição corporal foi avaliada no Laboratório de qualidade de alimento (LQA), na Universidade Estadual do Oeste do Paraná, Paraná, Brasil, com 16 peixes inteiros de cada tratamento, na qual foi realizada a secagem prévia das amostras biológicas em temperatura a $55^{\circ} \mathrm{C}$ durante 72 horas em estufa com ventilação forçada. Após a secagem, estas 
amostras passaram por uma moagem em moinho de martelo, e posteriormente foi realizada a análise de umidade, extrato etéreo, proteína bruta e matéria mineral, segundo as prescrições da Association of Official Analytical Chemists, AOAC (1995).

\subsection{Amostras biológicas}

No final do experimento três peixes de cada unidade experimental por tratamento foram anestesiados com eugenol diluído na água (100 mg.. $\left.\mathrm{L}^{-1}\right)$ para os procedimentos de coletas de amostras de sangue dos peixes, utilizando seringas de 1,0 mL banhadas com anticoagulante EDTA (3,0\%), seguindo as recomendações do Comitê de Ética no Uso de Animais (CEUA) da Universidade Estadual do Oeste do Paraná (UNIOESTE) de registro 41/20. O sangue foi coletado por meio de punção caudal, sendo armazenado em Eppendorf individual para cada indivíduo e duas lâminas por indivíduo foram empregadas para confecção do esfregaço do sangue para posteriores análises hematológicas (De Pádua et al., 2013). Além disso, foram retirados o intestino, fígado e a gordura visceral com materiais cirúrgicos (tesoura, pinça e bisturi), para serem mensurados peso e comprimento. Estes órgãos foram colocados em placa de Petri para avaliação dos índices somáticos (Fonseca et al., 2020).

O intestino e o fígado foram conservados em Alfac durante 24 horas de acordo com Caputo, Gitirana e Manso (2010) e depois transferidos para o álcool $70 \%$ até o momento do processamento histológico.

\subsection{Hematologia}

O número de eritrócitos foi contado através de uma câmara de Neubauer pelo método de hemocitômetro, utilizando-se o líquido de Hayem (1:200) em pipeta automática, sendo os valores obtidos utilizados para calcular o número total de leucócitos.

A contagem diferencial e total dos leucócitos foi feita através de duas lâminas por peixe de cada tratamento, realizado o esfregaço do sangue e em seguida coradas com corantes hematológicos com May-Grünwald Giemsa. A contagem e identificação das células foi realizada através de microscópio (P1 Olympus BX 50, Manila, Filipinas), na objetiva de 100 vezes e percorrendo toda a lâmina em "zig-zag" conforme descrito por Tavares-Dias e Moraes (2004). Para avaliação diferencial foram contadas 200 células, sendo estabelecido um percentual de linfócitos, neutrófilos e monócitos. Na contagem total foram contadas 2000 (duas mil) células e identificando o número de leucócitos.

Para realizar a análise bioquímica plasmática da glicose $\left(\mathrm{mg}_{\mathrm{aL}} \mathrm{dL}^{-1}\right)$ de cada peixe, as amostras foram centrifugadas a $2.500 \mathrm{rpm}$ por cinco minutos e depois conservadas em eppendorf em um freezer, para ser analisado com o uso de "kit de glicose" específicos (Gold Analisa Diagnóstica Ltda, Belo Horizonte - Minas Gerais, Brasil) através do método Enzimáticocolorimétrico, conforme as instruções do fabricante (Fonseca et al. 2020).

\subsection{Histologia}

As amostras dos intestinos e fígados foram preparados no Laboratório de Histologia do Gemaq, na UNIOESTE/Campus Toledo/PR. Amostras do intestino médio e do fígado foram desidratadas em série ascendente de álcoois (70, 80, 90,100 I, 100 II, 100\% III), diafanizadas em xilol, e colocadas em parafina histológica, para a realização dos cortes semisseriados de $7 \mu \mathrm{m}$. Para a coloração das lâminas do intestino e fígado foram preparadas uma lâmina por indivíduo, coradas com Hematoxilina-Eosina (HE), para as análises da morfologia dos vilos e morfometria dos núcleos dos hepatócitos.

As análises dos cortes histológicos foram realizadas em microscópio óptico (P1 Olympus BX 50, Manila, Filipinas), acoplado a câmera BEL Capture, utilizando as objetivas de 40x e 100x para as capturas de imagens dos cortes de intestino e fígado respectivamente. Um sistema de análises de imagens Eurekam 3.0 Plus foi utilizado para mensuração dos vilos e dos núcleos dos hepatócitos. 
A histomorfometria das vilosidades intestinais foi realizada medindo a altura de dez vilosidades (da base para o topo) e a largura das dobras (próximas à região do ápice) por amostra, e a espessura da túnica muscular (músculo liso) em dez pontos por amostra, usando as objetivas 40x e 100x. A histomorfometria foi realizada de acordo com Rodrigues et al. (2017), a partir de cinco fotos selecionadas aleatoriamente com uma ampliação de 1000x. A área $\left(\mu \mathrm{m}^{2}\right)$, perímetro $(\mu \mathrm{m})$, e o diâmetro $(\mu \mathrm{m})$ dos núcleos dos hepatócitos foram mensuradas em 50 células aleatoriamente por lâminas para calcular o volume do núcleo do hepatócito $\operatorname{Vnh}\left(\mu \mathrm{m}^{3}\right)=4 / 3 \pi \cdot \mathrm{r}^{3}$, onde r é o raio nuclear segundo (Strüssmann et al., 1990; Fonseca et al., 2020).

\subsection{Análise Estatística}

Os dados obtidos foram submetidos aos testes de normalidade e homocedasticidade, com à análise de variância unifatorial (ANOVA One-way). Posteriormente foi realizado o teste de comparação de médias, Teste de Tukey com níveis de significância de 5\%. Para os dados que não seguiram distribuição normal, foram realizadas as transformações (Log, Raiz e Rank) e todas foram realizadas utilizando-se o Software STATISTICA 7.0 (Carvalho et al., 2019).

\section{Resultados e Discussão}

Foram registradas às variáveis físico-químicas da água, de temperatura $25 \pm 1^{\circ} \mathrm{C} ; \mathrm{pH} 7,0 \pm 1,0$; oxigênio dissolvido $5,0 \pm 1,0 \mathrm{mg} . \mathrm{L}^{-1}$; sólidos totais dissolvidos $223 \pm 98 \mathrm{mg} . \mathrm{L}^{-1} \mathrm{e}$ a condutividade elétrica $352 \pm 173 \mu \mathrm{S} . \mathrm{cm}^{-1}$. Entre os tratamentos e ao longo do experimento não apresentaram diferenças $(p>0,05)$ que pudessem interferir nos resultados obtidos, pois os mesmos permaneceram de acordo com a faixa ideal recomendada para produção de peixes de clima tropical (Boyd, 1990; SipaúbaTavares, 1995).

Analisando o desempenho zootécnico da tilápia do Nilo, não houve diferença ( $\mathrm{p}>0,05)$ no peso médio final, no ganho de peso e comprimento do intestino (Tabela 2). Guimarães et al. (2019) testando B. subtilis e Lactobacillus plantarum em larvas de tilápia do Nilo durante a fase de inversão sexual, não constaram efeito significativo no desempenho, os mesmos autores relataram que alguns probióticos para demonstrar os seus efeitos benéficos requerem um agente estressor como, má qualidade de água, presença de enfermidade no cultivo ou superprodução em espaço reduzido. Porém Brito et al. (2019) ao testar cepas probióticas comercial B. subtilis, B. amyloliquefaciens, B. mycoides, megatherium por 30 dias em juvenis de tilápias do Nilo, com desafio sanitário, não encontraram resultados que pudessem expressar diferenças estatísticas no ganho de peso entre os tratamentos. Porém em um trabalho realizado por Aly et al. (2008) ao utilizar probiótico comercial (B. pumilus e Organic Green TM) no cultivo de tilápias do Nilo submetidas a infecção por Aeromonas hydrophila $\left(10^{8}\right.$ bactérias $\left.\mathrm{mL}^{-1}\right)$, por 2 meses de cultivo obteve resultado significativo no ganho de peso.

A sobrevivência dos juvenis de tilápia do Nilo não foi diferente $(p>0,05)$ entre os tratamentos, assim como a taxa de crescimento especifico. Esses resultados estão de acordo com os encontrados por Albuquerque (2011) ao adicionar na alimentação probióticos para juvenis de tilápia do Nilo, linhagem Gift e Azevedo et al., (2016) ofertando prebiótico, probiótico e simbiótico para juvenis de tambaqui em duas densidades de estocagem. No entanto Ramirez et al. (2016) ao testar o efeito da inclusão de probióticos microencapsulado (B. megaterium, B. polymyxa e Lactobacillus delbueckii) em tilápia vermelha (Oreochromis sp.), em concentração de $1,2 \times 10^{5} \mathrm{UFC} / \mathrm{g}$, encontraram resultados positivos $(\mathrm{p}<0,05)$ para taxa de sobrevivência e taxa de crescimento especifico, o mesmo ainda afirma que o uso de microorganismos endógenos melhora o desempenho zootécnico em peixes. A adição do complexo de bactérias e $B$. subtilis também não influenciou $(\mathrm{p}>0,05)$ os índices hepatosomático e viscerosomatico entre os tratamentos (Tabela 2). 
Tabela 2. Desempenho zootécnico de juvenis de tilápia do Nilo (Oreochromis niloticus) submetida ao complexo de bactérias e Bacillus subtilis.

\begin{tabular}{|c|c|c|c|c|}
\hline \multirow[b]{2}{*}{ Variáveis } & \multicolumn{3}{|c|}{ Tratamentos } & \multirow[b]{2}{*}{ Valor-p } \\
\hline & $\mathbf{C}$ & CB & $\mathbf{B S}$ & \\
\hline PMI (g) & $4,87 \pm 0,64$ & $4,91 \pm 0,80$ & $5,09 \pm 0,29$ & NS \\
\hline PMF (g) & $22,83 \pm 4,90$ & $23,85 \pm$ & $21,40 \pm 3,81$ & NS \\
\hline CI (cm) & $34,60 \pm 6,50$ & $36,99 \pm 6,97$ & $32,45 \pm 7,86$ & NS \\
\hline SO $(\%)$ & $81,25 \pm 16,13$ & $78,12 \pm 27,71$ & $68,75 \pm 16,13$ & NS \\
\hline $\operatorname{CAA}(\mathrm{g})$ & $1,62 \pm 0,39$ & $1,98 \pm 0,84$ & $1,99 \pm 0,56$ & NS \\
\hline GP(g) & $17,95 \pm 5,14$ & $18,94 \pm 1,37$ & $16,31 \pm 3,82$ & NS \\
\hline EA & $0,10 \pm 0,02$ & $0,09 \pm 0,01$ & $0,09 \pm 0,01$ & NS \\
\hline TCE (\%) & $2,83 \pm 0,54$ & $2,93 \pm 0,16$ & $2,63 \pm 0,35$ & NS \\
\hline IVS (\%) & $4,88 \pm 0,63$ & $5,06 \pm 1,54$ & $4,23 \pm 0,43$ & NS \\
\hline IHS (\%) & $1,56 \pm 0,67$ & $1,53 \pm 0,17$ & $2,08 \pm 0,70$ & NS \\
\hline
\end{tabular}

Valores apresentados como média \pm desvio padrão para dados normalmente distribuídos. $\mathrm{C}=$ tratamento controle; $\mathrm{CB}=$ adição de complexo de bactérias; $\mathrm{BS}$ = adição de probiótico $B$. subtilis; $\mathrm{NS}=$ não houve diferença significativa; *= diferença significativa $(\mathrm{p}<0,05)$ pelo teste de Tukey. Peso Inicial (PMI); Peso Final (PMF); Comprimento do intestino (CI); Sobrevivência (SO); Conversão Alimentar Aparente (CAA); Ganho de Peso (GP); Eficiência Alimentar (EA); Taxa de Crescimento Específico (TCE); Índice Viscerossomatico (IVS): Índice Hepatossomatico (IHS)

Fonte: Dados oriundos da pesquisa-

A inclusão de probióticos comercial adicionados à dieta para juvenis de tilápia do Nilo, não influenciou,-na composição centesimal para umidade, proteína bruta, lipídios totais e matéria mineral (Tabela 3). Este trabalho está de acordo com o encontrado por Albuquerque et al. (2015) que também não observaram diferenças para proteína bruta, extrato etéreo, umidade e matéria mineral ao adicionar dois produtos comerciais B. subtilis C-3102 e B. cereus var. Toyoi em juvenis de tilápia, variedade Gift. Logo Schwarz et al. (2016) encontraram diferenças, somente para proteína bruta na composição corporal em juvenis de tilápia do Nilo com a inclusão de 0,25\% levedura Saccharomyces cerevisiae.

Tabela 3. Composição centesimal de juvenis de tilápia do Nilo (Oreochromis niloticus) durante 53 dias de experimento submetida ao complexo de bactérias e Bacillus subtilis.

\begin{tabular}{lccccc}
\hline & & \multicolumn{3}{c}{ Tratamentos } \\
Variáveis & Inicial & C & CB & BS & Valor-p \\
\cline { 3 - 6 } Umidade (\%) & $79,52 \pm 0,02$ & $76,46 \pm 0,02$ & $76,76 \pm 0,04$ & $76,05 \pm 0,005$ & NS \\
Proteína bruta (\%) & $11,82 \pm 0,02$ & $12,45 \pm 0,15$ & $12,21 \pm 0,35$ & $13,16 \pm 0,22$ & NS \\
Extrato etéreo (\%) & $5,13 \pm 0,08$ & $5,79 \pm 0,10$ & $5,51 \pm 0,17$ & $5,67 \pm 0,10$ & NS \\
Matéria mineral (\%) & $3,40 \pm 0,08$ & $3,58 \pm 0,06$ & $3,62 \pm 0,01$ & $3,79 \pm 0,02$ & NS \\
\hline
\end{tabular}

Valores apresentados como média \pm desvio padrão para dados normalmente distribuídos. $\mathrm{C}=$ tratamento controle; $\mathrm{CB}=$ adição de complexo de bactérias; BS = adição de probiótico B. subtilis; NS= não houve diferença significativa pelo teste de Tukey.

Fonte: Dados oriundos da pesquisa.

Nenhum efeito foi verificado nos números de leucócitos, linfócitos, neutrófilos e monócitos. Além disso, para concentração de glicose também não foi observado diferença estatística significativas (Tabela 4). No entanto para os números de eritrócitos a análise de variância evidenciou efeito significativo $(\mathrm{p}<0,05)$ entre os tratamentos. Para o tratamento $\mathrm{CB}$ foi 
observado maiores números de eritrócitos do que nos grupos C e BS, porém não é possível afirmar causa ou implicação dessa diferença entre os dois grupos tratados com complexo de bactérias e B. subtilis. Porém Costa et al. (2014) observou valores superiores ao do presente trabalho, ao adicionar diferentes fontes de óleos na alimentação de tilápia do Nilo. Contudo Wang et al. (2017) observaram melhora no estado imunológico em tilápias do Nilo, após serem tratados com $B$. cereus na água e na ração. Entretanto, neste presente estudo não houve diferenças nos demais parâmetros sanguíneos.

Tabela 4. Parâmetros hematológicos de juvenis de tilápia do Nilo (Oreochromis niloticus) submetidos ao complexo de bactérias e Bacillus subtilis.

\begin{tabular}{|c|c|c|c|c|}
\hline \multirow[b]{2}{*}{ Variáveis } & \multicolumn{3}{|c|}{ Tratamentos } & \multirow[b]{2}{*}{ Valor-p } \\
\hline & C & CB & $B S$ & \\
\hline Eritrócitos $\left(10^{6} \mu \mathrm{L}-1\right)$ & $2,70 \pm 2,00 \mathbf{a b}$ & $3,30 \pm 1,50 \mathbf{a}$ & $1,60 \pm 5,20 \mathbf{b}$ & $*$ \\
\hline Leucócitos $\left(10^{4} \mu \mathrm{L}-1\right)$ & $2,60 \pm 2,11$ & $2,50 \pm 1,16$ & $1,50 \pm 5,12$ & NS \\
\hline Linfócitos $\left(10^{4} \mu \mathrm{L}^{-1}\right)$ & $2,59 \pm 2,10$ & $2,40 \pm 1,15$ & $1,50 \pm 5,06$ & NS \\
\hline Monócitos $\left(10^{3} \mu \mathrm{L}-1\right)$ & $1,52 \pm 1,06$ & $2,52 \pm 1,33$ & $2,52 \pm 1,38$ & NS \\
\hline Neutrófilos $\left(10^{3} \mu \mathrm{L}-1\right)$ & $2,04 \pm 1,41$ & $2,75 \pm 1,50$ & $3,10 \pm 2,18$ & NS \\
\hline Glicose (mg. dL $\left.\mathbf{L}^{-1}\right)$ & $195,07 \pm 102,43$ & $162,64 \pm 54,34$ & $144,83 \pm 57,62$ & NS \\
\hline
\end{tabular}

Dados apresentados como média \pm desvio padrão para dados normalmente distribuídos. $\mathrm{C}=$ tratamento controle; $\mathrm{CB}=$ adição do complexo de bactérias; $\mathrm{BS}=$ adição de $B$. subtilis; NS= não houve diferença significativa; *= diferença significativa $(\mathrm{p}<0,05)$ pelo teste de Tukey. Fonte: Dados oriundos da pesquisa.

A análise do intestino revelou diferença significativa na altura e largura dos vilos do tratamento com B. subtilis em relação ao complexo de bactérias e controle, mas para a espessura da túnica o complexo de probiótico teve o maior valor médio em relação ao que recebeu B. subtilis (Tabela 5). Segundo Azevedo et al. (2016) a capacidade de absorção do intestino está ligada ao tamanho e o número das vilosidades, sendo essas estruturas podem ser modificadas por fatores antinutricionais ou microorganismos, evidenciando um desequilíbrio e alteração nos vilos.

As vilosidades são estruturas que podem sofrer alterações em sua morfologia e histologia, em função dos ingredientes que compõem a dieta (Schwarz et al., 2011). Essas alterações no comprimento das vilosidades e larguras do vilos, irá proporcionar melhor absorção de nutrientes (Caspary, 1992; Batista et al., 2016).

Em estudo realizado por Mello et al. (2013) foi observado que o incremento de $4.0 \mathrm{~g} \mathrm{~kg}^{-1}$ de $B$. cereus e $B$. subtilis na dieta de juvenis de tilápia do Nilo, ocasionando aumento da altura e largura das vilosidades, o que aumentou a área de absorção. Resultados significativos para altura dos vilos e espessura do epitélio também foram encontrados por (Carvalho et al., 2011) ao adicionar pré (0,5\% de MOS), e probióticos (4,15 X $10^{7}$ UFC de B. subtilis) na alimentação de juvenis de tilápias do Nilo. Silva (2014), Reda e Selim (2015) também perceberam mudanças nas vilosidades ao adicionarem probiótico em ração comercial para tilápia do Nilo cultivada em tanque-rede. Fonseca et al. (2020), identificaram um aumento da altura dos vilos e na espessura da túnica intestinal de tilápia do Nilo alimentados com complexo de probiótico, obtendo importantes resultados imunológico para tilápia do Nilo. De acordo com Jesus (2014), o crescimento da morfometria intestinal mostra a capacidade dos probióticos na diminuição da adesão de bactérias patogênicas do epitélio intestinal. Por outro lado, o aumento da túnica está relacionado à adaptação da estrutura intestinal em resposta à adição probiótica (Rotta, 2003).

O tempo de alimentação, os tipos de microorganismos utilizado e a quantidade administradas, são fatores que interferem no sucesso do uso do probiótico para a dieta (Khojasteh, 2012; Ramos et al., 2017). Neste estudo foi observado uma túnica mais espessa no tratamento com complexo de bactérias, diferente dos tratamentos com B. subtilis e o controle. Destaca-se que para esse estudo, os animais utilizados, não foram submetidos a desafios. Porém, foi constatado uma 
diferença significativa na morfologia do intestino, isso não foi o suficiente para alterar dados de desempenho zootécnico, ou parâmetros imunológicos.

Tabela 5. Parâmetros morfológicos do intestino de juvenis de tilápia do Nilo (Oreochromis niloticus) submetida à complexo de bactérias e Bacillus subtilis.

\begin{tabular}{lcccc}
\hline & \multicolumn{3}{c}{ Tratamentos } & Valor-p \\
\cline { 2 - 4 } Variáveis & $\mathbf{C}$ & $\mathbf{C B}$ & $\mathbf{B S}$ & $*$ \\
Altura dos vilos $(\boldsymbol{\mu m})$ & $183,19 \pm 76,41 \mathbf{a b}$ & $112,35 \pm 20,81 \mathbf{b}$ & $209,19 \pm 72,42 \mathbf{a}$ & $*$ \\
Largura dos vilos $(\boldsymbol{\mu m})$ & $90,34 \pm 27,60 \mathbf{b}$ & $42,57 \pm 7,52 \mathbf{c}$ & $94,83 \pm 11,40 \mathbf{a}$ & $*$ \\
Espessura da túnica $(\boldsymbol{\mu m})$ & $20,90 \pm 8,92 \mathbf{c}$ & $30,07 \pm 4,75 \mathbf{a}$ & $21,18 \pm 3,21 \mathbf{b}$ & \\
\hline
\end{tabular}

Valores apresentados como média \pm desvio padrão para dados normalmente distribuídos. $\mathrm{C}=$ tratamento controle; $\mathrm{CB}=$ adição de complexo de bactérias; BS = adição de $B$. subtilis; *= houve diferença significativa pelo teste de Tukey.

Fonte: Dados oriundos da pesquisa.

Nas análises morfometrias dos núcleos dos hepatócitos de tilápia do Nilo, o tratamento que continha complexo de bactérias, obteve maiores valores para as variáveis mensuradas, área $\left(\mu \mathrm{m}^{2}\right)$, perímetro $(\mu \mathrm{m})$ e volume $\left(\mu \mathrm{m}^{3}\right)$ dos núcleos dos hepatócitos apresentaram diferença significativa $(p<0,05)$ entre os tratamentos (Tabela 6).

Tabela 6. Característica morfométrica dos núcleos dos hepatócitos de juvenis de tilápia do Nilo (Oreochromis niloticus) submetida ao complexo de bactérias e Bacillus subtilis.

\section{Tratamentos}

\begin{tabular}{lcccc}
\cline { 2 - 4 } Variáveis & $\mathbf{C}$ & $\mathrm{CB}$ & BS & Valor-p \\
Perímetro do Núcleo $(\mu \mathrm{m})$ & $8,49 \pm 0,53 \mathbf{b}$ & $8,96 \pm 0,69 \mathbf{a}$ & $8,01 \pm 0,71 \mathrm{c}$ & $*$ \\
Área do Núcleo $\left(\mu \mathrm{m}^{2}\right)$ & $18,08 \pm 2,26 \mathbf{b}$ & $20,14 \pm 3,06 \mathbf{a}$ & $15,40 \pm 2,11 \mathrm{c}$ & $*$ \\
Volume do núcleo $\left(\mu \mathrm{m}^{3}\right)$ & $41,21 \pm 7,44 \mathbf{b}$ & $47,97 \pm 10,63 \mathbf{a}$ & $34,48 \pm 9,43 \mathbf{c}$ & $*$
\end{tabular}

Valores apresentados como média \pm desvio padrão para dados normalmente distribuídos. $\mathrm{C}=$ tratamento controle; $\mathrm{CB}=$ adição de complexo de bactérias; $\mathrm{BS}=$ adição de probiótico $B$. subtilis; *= houve diferença significativa pelo teste de Tukey.

Fonte: Dados oriundos da pesquisa.

Fonseca et al. (2020) citam que em suas analises observaram maiores áreas de núcleos de hepatócitos após alimentação com probióticos, essas alterações são explicadas pelo manejo nutricional aplicado (Rodrigues et al., 2017). Segundo Ostaszewska et al. (2011) o aumento do tamanho do núcleo dos hepatócitos depende da quantidade de glicogênio ou lipídios armazenados no citoplasma, revelando alterações no metabolismo e o aparecimento de necrose hepática ou esteatose Caballero et al. (2004) Raskovic et al. (2011). Estes autores afirmaram que proteína na dieta em excesso, necessitam de maiores níveis de energia para serem metabolizadas, e a parte não metabolizada se concentra em forma de gordura no fígado, sobrecarregando suas funções, conforme destacado por Almeida et al. (2011).

Já Rodrigues et al. (2017) constataram a redução no metabolismo dos hepatócitos, ao submeter híbridos de surubim (Pseudoplatystoma reticulatum $\times$ Pseudoplatystoma corruscans) a uma dieta inadequada, e por consequência a diminuição da área do núcleo. Da mesma forma Cabalerro et al. (2004) viu alterações histológicas ao aumentar níveis de óleo de soja em rações para "sea bream" (Sparus aurata L.), resultando no aparecimento de esteatose nos hepatócitos. Porém Lins Rodrigues $e t$ al. (2020) observaram uma redução nos parâmetros morfométricos, avaliados pelo uso de glicogênio e estrutura de hepatócitos, 
quando os peixes foram alimentados com probióticos, essa redução tira a sobrecarga no fígado durante os processos metabólicos (Power et al., 2000).

O fígado é fundamental para o metabolismo dos nutrientes e nos dá respostas do estado nutricional em peixes, essas alterações podem ser resultado de uma dieta desbalanceada, exposição a substâncias químicas ou drogas, respondendo com adaptações, lesão ou morte celular (Bolla et al., 2011; Raskovic et al., 2011; Honorato et al., 2014). Neste estudo realizado, não foi observado comprometimento das células hepáticas mesmo apresentando aumento na estrutura dos hepatócitos em um dos grupos tratados.

\section{Conclusão}

Os resultados mostraram que não diferenças entre os tratamentos em relação ao desempenho zootécnico, mas houve melhoras em relação à morfologia do intestino, o que pode evidenciar melhoras no estado nutricional dos animais.

\section{Agradecimentos}

Os autores agradecem à Universidade Estadual do Oeste do Paraná (UNIOESTE) e ao Grupo de Estudos em Gestão da Aquicultura (Gemaq) por suas contribuições, em relação a infraestrutura laboratorial, que foram essenciais para o desenvolvimento deste estudo. $\mathrm{O}$ agradecimento se estende à CAPES pela cessão da bolsa de estudos na modalidade Demanda Social. Os autores também agradecem à empresa Biotecnal por fornecer o probiótico utilizado neste estudo.

\section{Referências}

Almeida, L. C., Avilez, I. M., Honorato, C. A. \& Moraes, G. (2011). Gowth and metabolic responses of tambaqui (Colossoma macropomum) fed dietslevel of protein and lipd. Aquaculture Nutrition, 17, 283-262.

Albuquerque, D. M., Marengoni, N. G., Mahl, I., Moura, M. C., Rodriguez-Rodriguez, M. D. P. \& Ribeiro, R. P. (2015). Bacillus cereus var. toyoi e Bacillus subtilis C-3102 em dietas para alevinos de tilápia do Nilo, linhagem GIFT. Bioscience Journal, 31(2), 532-540.

Aly, S. M., Mohamed, M. F. \& John, G. (2008). Effect of probiotic on the survival, growth and challenge infection in tilapia nilotica (Oreochromis niloticus) Aquaculture Research, 39(6), 647-656.

Amarante, J. F., Kolling, L., Ferronato, A. I., Vargas, A. C., Costa, M. M. \& Amarante, T. A. B. (2018). Resistência aos antimicrobianos de bactérias obtidas de carpas (Cyprinus carpio) cultivadas em sistema semi-intensivo. Ciência Animal Brasileira, 19(1), 1-7.

PEIXE BR. Anuário Brasileiro da Piscicultura Peixes BR 2021. Associação Brasileira da Piscicultura, https://www.peixebr.com.br/ anuario-2021.

Azevedo, R. V., Fosse Filho, J. C., Pereira, S. L., Cardoso, L. D., Júnior, M. V. V. \& Andrade, D. R. (2016). Suplementação com prebiótico, probiótico e simbiótico para juvenis de tambaqui a duas densidades de estocagem. Pesquisa Agropecuária Brasileira, 51(1), 9-16.

Bolla, S., Nicolaisen, O. \& Amin, A. (2011). Liver alterations induced by long term feeding on commercial diets in Atlantic halibut (Hippoglossus hippoglossus L.) female. Histological and biochemical aspects. Aquaculture, 312(1-4), 117-125.

Boyd, C. E. (1990). Water quality in ponds for Aquaculture Alabama. Alabama, USA: Agricultural Experiment Station, Auburn University.

Brito, J. M., Ferreira, A. H. C., Júnior, H. A. S., Oliveira, A. P. A., Santos, C. H. L. \& Oliveira, L. T. S. (2019). Desempenho zootécnico de juvenis de tilápias do Nilo (Oreochromis niloticus) alimentados com cepas probióticas e submetidos a desafio sanitário. Ciência Animal Brasileira, $20,1-9$.

Batista, S, Medina, A., Pires, M. A., Morinigo, M. A., Sansuwan, K., Fernandes, J. M. O, Valente, L. M. P. \& Ozório, R. O. A. (2016). Innate immune response, intestinal morphology and microbiota changes in Senegalese sole fed plant protein diets with probiotics or autolysed yeast. Applied microbiology and biotechnology, 100(16), 7223-7238.

Caballero, M. J., Izquierdo, M. S., Kjøsvik, E., Fernández, A. J. \& Rosenlund, G. (2004). Histological alterations in the liver of sea bream, Sparus aurata L. caused by short-or long-term feeding with vegetable oils. Recovery of normal morphology after feeding fish oil as the sole lipid source. Journal of Fish Diseases, 27, 531-541.

Caputo, L. F. G., Gitirana, L.B. \& Manso, P. P. A. (2010). Técnicas histológicas. In: Molinaro, E. M., Caputo, L. F. G. \& Amendoeira, M. R. R. Conceitos e métodos para formação de profissionais em laboratórios de saúde. Rio de Janeiro: Escola Politécnica de Saúde Joaquim Venâncio.

Carvalho, J.V., Lira, A. D., Costa, D. S. P., Moreira, E. L. T., Pinto, L. F. B., Abreu, R. D. \& Albinati, R. C. B. (2011). Desempenho zootécnico e morfometria intestinal de alevinos de tilápia-do -Nilo alimentados com Bacillus subtilis ou mananoligossacarídeo. Revista Brasileira de Saúde Produção Animal, 12, 176187. 
Carvalho, K. V., Luczinski, T. G., Boscolo, W. R., de Freitas, J. M. A. \& Signor, A. (2020). Poultry byproducts and swine liver used in diets for Nile tilapia juveniles. Latin american journal of aquatic research, 48(5), 895-900.

Caspary W. F. (1992). Physiology and pathophysiology of intestinal absorption. The American Journal of Clinical Nutrition. 55, 299-308.

Costa, D. V. D., Ferreira, M. W., Navarro, R. D., Rosa, P. V. \& Murgas, L. D. S. (2014). Parâmetros hematológicos de tilápias-do-Nilo (Oreochromis niloticus) alimentadas com diferentes fontes de óleo. Revista Brasileira de Saúde e Produção Animal, 15(3), 754-764.

Da Costa, M. M., Peixoto, R. D. M., De Lima, C., Boijink, L. C., Meurer, F. \& De Vargas, A. C. (2008). Sensibilidade antimicrobiana de bactérias isoladas de Jundiá (Rhamdia quelen). Pesquisa Veterinária Brasileira, 28(10), 477-480.

De Brito, J. M., Ferreira, A. H. C., Junior, A., Araripe, M., Lopes, J., Duarte, A. \& Rodrigues, V. (2014). Probióticos, prebióticos e simbióticos na alimentação de não-ruminantes, revisão. Revista Eletrônica Nutritime, 11(1), 3070-3084.

De Pádua, S. B., Paiva, M. J. R. \& Dias-Tavares, M. Métodos para análise hematológica em peixes. Editora da Universidade Estadual de Maringá-EDUEM, 2013.

FAO. (2018). The State of World Fisheries and Aquaculture 2018-Meeting the sustainable development goals.

Ferreira, A. H. C., Brito, J. M. D., Lopes, J. B., Santana Júnior, H. A. D., Batista, J. M. M., Silva, B. R. \& Amorim, I. L. D. S. (2015). Probiótico na alimentação de pós-larvas de tilápias do Nilo submetidas a desafio sanitário. Revista Brasileira de Saúde e Produção Animal, 16(2), 430-43.

Guimaraes, M. C., De Carla Dias, D., De Araujo, F. V. A. P., Ishikawa, C. M. \& Tachibana, L. (2019). Probiotic Bacillus subtilis and Lactobacillus plantarum in diet of Nile tilapia. Boletim do Instituto de Pesca, 45(1).

Gutiérrez Ramirez, L. A., David Ruales, C. A., Montoya Campuzano, O. I. \& Betancur Gonzalez, E. (2016). Efecto de la inclusión en la dieta de probióticos microencapsulados sobre algunos parámetros zootécnicos en alevinos de tilápia roja (Oreochromis sp.). Revista de Salud Animal, 38(2), 112-119.

Honorato, C. A., Cruz, C. D., Carneiro, D. J., Machado, M. R., Nascimento, C. A. \& Saturnino, K. C. (2014). Histologia do fígado de tilápia do Nilo (Oreochromis niloticus) alimentados com dietas contendo silagem biológica de pescado. Pesquisa Veterinaria Brasileira, 34, 64-68.

IBGE, Produção da Pecuária Municipal 2018, Rio de Janeiro: IBGE, 2019. https://www.ibge.gov.br/estatisticas/economicas/agricultura-e-pecuaria/9107 producao-da-pecuaria-municipal.html.

IBGE. Produção da Pecuária Municipal, 2017. https://biblioteca.ibge.gov.br/visualizacao/periodicos/84/ppm_2017_v45_br_informativo. pdf

Ibrahem, M. D. (2015). Evolution of probiotics in aquatic world: potential effects, the current status in Egypt and recent prospectives. Journal of advanced research, 6(6), 765-791.

Jesus, G.F.A. (2014). Weissella cibaria e sua ação probiótica no trato intestinal de surubins híbridos. 90f. (Dissertação de Mestrado, Universidade Federal de Santa Catarina-UFSC).

Fonseca, J. R. S., Carvalho, K. V., Da Silva, A. F. C., De Freitas, J. M. A., Signor, A. \& Feiden, A. (2020). Effects of Bac-Trat ${ }^{\circledR}$ Probiotic Complex on Growth, Hematological and Intestinal Parameters of Nile Tilapia, Reared at low Temperatures. Boletim do Instituto de Pesca, 46(2).

Khati, A., Chauhan, R. S., Nazir, I. \& Arya, P. (2018). Improved fish health: Key to succesful aquaculture. Journal of Entomology and Zoology Studies, 6(2), 898-902.

Khojasteh, S. M. B. (2012). The morphology of the post-gastric alimen-tary canal in teleost fishes: A brief review. International Journal of Aquatic Science, 3 , $71-88$.

Lins Rodigues, M., Damasceno, D. Z., Gomes, R. L. M., Sosa, B. D. S., Moro, E. B., Boscolo, W. R. \& Signor, A. (2021). Probiotic effects (Bacillus cereus and Bacillus subtilis) on growth and physiological parameters of silver catfish (Rhamdia quelen). Aquaculture Nutrition, 27(2), 454-467.

Mello, H. D., Moraes, J. R., Niza, I. G., Moraes, F. R. D., Ozório, R. O., Shimada, M. T., Engracia Filho, J. R. \& Claudiano, G. S. (2013). Efeitos benéficos de probióticos no intestino de juvenis de Tilápia-do-Nilo. Pesquisa Veterinária Brasileira, 33(6), 724-730.

Molina, G., Pelissari, F. M. \& Feirhmann, A. C. (2013). Consequências da desnutrição proteica para o trato gastrintestinal. Arquivos Do Museu Dinâmico Interdisciplinar, 13(1-2-3), 12-24.

Nakandakare, I. B., Iwashita, M. K. P., Dias, D. D. C., Tachibana, L., Ranzani-Paiva, M. J. T. \& Romagosa, E. (2013). Incorporação de probióticos na dieta para juvenis de tilápias-do-Nilo: parâmetros hematológicos, imunológicos e microbiológicos. Boletim do Instituto de Pesca, 39(2), 121-135.

Ostaszewska, T., Dabrowski, K., Kwasek, K., Verri, T., Kamaszewski, M., Sliwinski, J. \& Napora-Rutkowski, L. (2011). Effects of various diet formulations (experimental and commercial) on the morphology of the liver and intestine of 46 rainbow trout (Oncorhynchus mykiss) juveniles. Aquaculture Research, 42(12): 1796-1806.

Power, D. M., Melo, J. \& Santos R. A (2000). The effect of food deprivation and refeeding on the liver thyroid hormones and transthyretin in sea bream. Journal of Fish Biology, 56, 374-387.

Silva, D.J. \& Queiroz, A. C. (2002). Análise de Alimentos: métodos químicos ebiológicos. (3a Ed.), UFV. 235p.

Sibaúba-Tavares, L. H. 1995. Limnologia aplicada à aquicultura. Funep, 70.

Ramos, M. A., Batista, S., Pires, M. A., Silva, A. P., Pereira, L. F., Saavedra, M. J. \& Rema, P. (2017). Dietary probiotic supplementation improves growth and the intestinal morphology of Nile tilapia. animal, 11(8), 1259-1269. 
Research, Society and Development, v. 10, n. 8, e38210817568, 2021

(CC BY 4.0) | ISSN 2525-3409 | DOI: http://dx.doi.org/10.33448/rsd-v10i8.17568

Rašković, B. S., Stanković, M. B., Marković, Z. Z. \& Poleksić, V. D. (2011). Histological methods in the assessment of different feed effects on liver and intestine of fish. Journal of Agricultural Sciences, 56, 87-100.

Reda, R. \& Selim, K. (2015). Avaliação de Bacillus amyloliquefaciens na composição desempenho de crescimento, morfologia intestinal, hematologia e corpo de tilápia, Oreochromis niloticus. Aquicultura International, 21 (1), 203-217.

Rego, M., Silva, E., Calazans, N., Vogeley, J., Nery, R., Soares, R. \& Peixoto, S. (2012). Utilização de probiótico e antibiótico no cultivo de pós-larvas do camarão Branco Litopenaeus vannamei. Atlântica (Rio Grande), 34(2), 137-143.

Ribeiro, P. A. P., Costa, L. S. \& Logato, P. V. R. (2008). Probióticos na aquicultura. Revista Nutritime, 6(1), 837-846.

Rodrigues, R. A., Saturnino, K. C. \& Fernandes, C. E. (2017). Liver histology and hi tomorphometry in hybrid sorubim (Pseudoplatystoma reticulatum $\times$ Pseudoplatystoma corruscans) reared on intensive fish farming. Aquaculture Research, 48(9), 5083-5093.

Rotta, M.A. (2003). Aspectos da fisiologia e estrutura do sistema digestivo dos peixes relacionados à piscicultura. Embrapa Pantanal-Documentos (INFOTECA-E).

Schulter, E. P. \& Vieira Filho, J. E. R. (2017). Evolução da piscicultura no Brasil: Diagnóstico e desenvolvimento da cadeia produtiva de tilápia (No. 2328). Texto para Discussão.

Schwarz, K. K., Nascimento, J. C., Gomes, V. A. A., Silva, C. H., Salvador, J. G., Fernandes, M. R. \& Nunes, R. M. (2016). Desempenho zootécnico de alevinos de tilápias do Nilo (Oreochromis niloticus) alimentados com levedura de Saccharomyces cerevisiae. Holos, 3 (1), 104-113.

Silva, T. F. A., Petrillo, T. R., Yunis-Aguinaga, J., Marcusso, P. F., Da Silva Claudiano, G., De Moraes, F. R. \& De Moraes, J. R. E. (2015). Effects of the probiotic Bacillus amyloliquefaciens on growth performance, hematology and intestinal morphometry in cage-reared Nile tilapia. Latin American Journal of Aquatic Research, 43(5), 963-971.

Sipaúba -Tavares, L. H. S. (1995). Limnoligia aplicada à aquicultura. Funep.

Strüssmann, C. A. \& Takashima, F. (1990). Hepatocyte nuclear size and nutritional condition of larval pejerrey, Odontesthes bonariensis (Cuvier et Valenciennes). Journal of Fish Biology, 36(1), 59-65.

Van Hai, N. (2015). Research findings from the use of probiotics in tilapia aquaculture: a review. Fish and Shellfish Immunology, 45(2), 592-597.

Wang, M., Liu, G., Lu, M., Ke, X., Liu, Z., Gao, F. \& Yu, D. (2017). Effect of Bacillus cereus as a water or feed additive on the gut microbiota and immunological parameters of Nile tilapia. Aquaculture Research, 48(6), 3163-3173. 